\title{
Lidil
}

Revue de linguistique et de didactique des langues

\section{Lire des textes littéraires hybrides puis écrire son texte en L2: quelle place pour l'image et pour les « langues en réserve » de l'apprenant?}

Reading Hybrid Literary Texts As a Stimulus to Writing in L2: Student Recourse to the Image and "Languages in Reserve"

\section{Christèle Maizonniaux}

\section{OpenEdition}

\section{Journals}

Édition électronique

URL : http://journals.openedition.org/lidil/4897

DOI : $10.4000 /$ lidil.4897

ISSN : 1960-6052

\section{Éditeur}

UGA Éditions/Université Grenoble Alpes

\section{Édition imprimée}

ISBN : 978-2-37747-048-8

ISSN : 1146-6480

\section{Référence électronique}

Christèle Maizonniaux, «Lire des textes littéraires hybrides puis écrire son texte en L2 : quelle place pour l'image et pour les « langues en réserve » de l'apprenant ? », Lidil [En ligne], 57 | 2018, mis en ligne le 01 mai 2018, consulté le 01 mai 2019. URL : http://journals.openedition.org/lidil/4897 ; DOI : 10.4000/lidil.4897

Ce document a été généré automatiquement le 1 mai 2019.

(C) Lidil 


\section{Lire des textes littéraires hybrides puis écrire son texte en L2 : quelle place pour l'image et pour les « langues en réserve » de l'apprenant?}

Reading Hybrid Literary Texts As a Stimulus to Writing in L2: Student Recourse to the Image and "Languages in Reserve"

\section{Christèle Maizonniaux}

\section{Introduction}

1 Au sein du champ littéraire francophone, l'écriture autobiographique relatant l'exil et le voyage est un domaine particulièrement riche, déjà exploité en contexte universitaire australien dans le cadre de l'enseignement du français langue étrangère (Edwards \& Hogarth, 2016; Crozet, 2014). Cependant, l'orientation didactique varie. Tous les didacticiens ne proposent pas aux apprenants de pratiquer l'écriture individuelle autobiographique.

2 Malgré la diversité qui caractérise les parcours linguistiques et culturels de nombreux apprenants en classes de langue étrangère, rares sont les démarches qui, dans le cadre de production d'écrits en contexte universitaire, invitent les apprenants à recourir aux langues de leur répertoire ou à faire montre de créativité (Maizonniaux, à paraitre). En outre, les langues et cultures d'origine sont souvent invisiblisées dans le contexte de l'enseignement supérieur en Australie (voir Diallo \& Maizonniaux, 2017 ; Liyanage, Singh \& Walker, 2016).

3 Inspirée par les travaux de plusieurs chercheuses travaillant dans le domaine des ateliers d'écriture multilingues en classe de langue (Mathis, 2014; Dompmartin-Normand \& 
Le Groignec, 2015), nous avons repris un travail élaboré antérieurement visant la lectureécriture à partir d'un corpus de textes autobiographiques sur la migration et y avons incorporé des textes ou iconotextes multilingues (BD, livre pour la jeunesse). L'objectif était de susciter la créativité chez les apprenants et d'inviter ceux-ci à imiter certaines des techniques observées dans les ouvrages étudiés ou à proposer des démarches originales. Nous faisions l'hypothèse que les supports proposés permettraient de répondre aux besoins et attentes des apprenants dans leur diversité et les inviteraient à intégrer les langues de leur répertoire et la dimension visuelle ou graphique dans leurs écrits créatifs.

4 Cet article présente les résultats d'une démarche exploratoire mise en place en 2014 dans une université australienne. Nous nous pencherons sur les productions pour analyser les éventuelles traces d'hybridité présentes, qu'il s'agisse de multilinguisme ou de multimodalité, pour en questionner la forme, la fréquence et le lien avec les techniques et styles observés dans les documents étudiés.

\section{2. Écriture de soi et écriture multilingue dans l'enseignement-apprentissage des langues}

\subsection{L'écriture de soi en contexte scolaire et universitaire}

5 Je retiens, à la suite de Bishop et Penloup (2006), « comme relevant de l'écriture de soi, les situations d'écriture à la première personne qui sollicitent explicitement le vécu des élèves » (p. 7). En outre, " est écriture de soi, alors, une écriture qui manifeste dans des marques linguistiques, énonciatives, lexicales [...] une posture d'affirmation de la subjectivité. Cette posture s'accompagne d'éléments textuels qui indiquent que le narrateur a choisi d'ancrer son récit dans sa propre histoire » (p. 6).

Bishop et Penloup (2006) soulignent que « dans la situation scolaire, l'élève doit composer avec, d'un côté, la contrainte scolaire qui impose le respect de la consigne et la démonstration d'une compétence rédactionnelle et, de l'autre, avec l'intention qui préside à l'écriture de soi » (p. 7). Chez l'apprenant plurilingue, cette double contrainte s'accompagne de la nécessité de composer avec les compétences qu'il a développées dans plusieurs littératies ou formes de littératies (voir Moore, 2006, p. 118).

7 Lejeune (2001) note dans un article consacré à l'écriture autobiographique en classe de français dans le secondaire en France à quel point ce type d'exercice est inhabituel. Il l'est tout autant en classe de français langue étrangère, même si, en contexte universitaire australien ou anglo-saxon, des cours sont proposés dans certains départements d'anglais qui sont uniquement dédiés à l'écriture de soi ou « life writing » (voir Cardell \& Douglas, 2018).

8 La biographie langagière, fréquemment utilisée dans la formation des futurs enseignants de langue dans le but de faire réfléchir ceux-ci sur leur expérience d'apprenants de langues, sur les langues de leur répertoire ou sur leur rapport à ces langues (voir Baroni \& Bemporad, 2011), est un exercice intéressant dans le cadre de l'écriture de soi. Non seulement elle convoque mais met en valeur les « langues en réserve ». 


\subsection{L'écriture multilingue en classe de langue}

9 Ces dernières années, plusieurs chercheurs dans le champ de la sociolinguistique et de la didactique des langues s'intéressent à l'écriture multilingue. En France, par exemple, des expériences ont lieu en contexte universitaire sous forme d'ateliers d'écriture, dans le cadre de l'enseignement du français langue étrangère (Dompmartin-Normand \& Le Groignec, 2015 ; Mathis, 2014, 2016) ou bien de l'anglais langue étrangère (Schultze, 2014 ; Greaves \& Di Stefano, 2015). Les participants peuvent être des apprenants qui viennent de s'installer en France. Le plus souvent, il s'agit d'apprenants de niveau B2-C1. Parfois, il s'agit d'expériences conduites auprès de grands groupes, certains évoquent une quarantaine d'étudiants (Schultze, 2014).

10 Le plus souvent, les démarches d'écriture multilingue sont en rapport avec la thématique de l'exil, du voyage. Il s'agit alors de produire des textes identitaires («identity texts ${ }^{1}$ ») qui « permettent aux apprenantes d'investir, de (dé) (re) construire et de valoriser leurs identités plurielles et de refléter de manière positive leurs expériences à travers les langues, parfois ambivalentes voire déstabilisantes» (Mathis, 2014). De manière récurrente, la lecture-découverte de textes multilingues précède les phases d'écriture puis de partage à l'intérieur du groupe d'apprentis-scripteurs. Les phases d'écriture sont le plus souvent précédées de "propositions d'écriture ", qui, pour Mathis (2014) sont "régulièrement négociées et négociables avec les participants du groupe »(p. 75). Elles peuvent, par exemple, inclure la rédaction d'une biographie langagière (Schultze, 2014), un travail sur les souvenirs (Mathis, 2014) ou encore une mise en voix des langues du répertoire des apprenants par le biais de marionnettes (Greaves \& Di Stefano, 2015). De manière intéressante, certains intègrent la variation linguistique dans leur démarche tant en ce qui concerne les supports écrits proposés qu'en ce qui concerne les options d'écriture possibles (Moïse, 2007). Dompmartin-Normand et Le Groignec (2015) insistent sur le hiatus existant entre les attentes des apprenants (essentiellement liées à l'amélioration des compétences linguistiques) et l'objectif des enseignants qui déplacent le curseur et visent «la progression d'habiletés transverses, le déplacement de représentations sur la difficulté de la langue, le rapport à l'écriture en langue "autre" et en général, dans sa dimension productive de mise en mots de soi et de son expérience »(p.61). Ces mêmes chercheuses mettent également en avant l'aspect inattendu, inhabituel de l'écriture multilingue.

11 En outre, la recherche met en exergue l'influence des textes sources sur les productions d'apprenants, certains qualifient d'ailleurs la première phase du travail consacrée à la lecture de phase « d'imprégnation / travail de compréhension des textes ou œuvres et de leurs cotextes » (Dompmartin-Normand \& Le Groignec, 2015), de «tremplin » (Mathis, 2016). Ces textes sources ont tout d'abord une fonction «inspirante» au sens où ils ouvrent des voies possibles dans l'écriture. Ils sont ensuite le lieu où des auteurs pratiquant l'écriture multilingue, "offrent une forme de légitimation à l'utilisation de plusieurs langues et variétés de langues que les apprenantes de langues n'utiliseraient probablement pas si le contexte didactique ne le permettait pas explicitement » (Mathis, 2016, p. 265).

$12 \mathrm{Au}$ niveau des résultats obtenus à la suite de la pratique de l'écriture multilingue, nombreux sont ceux qui signalent chez leurs apprenants le franchissement de «barrières ». L'écriture multilingue s'apparente encore à une "libération » : libération 
des carcans, libération par rapport à la norme, à un bien-écrire (Moïse, 2007), libération par rapport à des représentations axées sur le manque, l'imperfection (DompmartinNormand \& Le Groignec, 2015). Elle aboutit également à une révélation: en effet, « l'atelier leur permet de développer un faisceau d'habiletés reliées entre elles : celles de traducteur, d'expert de sa propre langue, d'expert de la langue autre, de lecteur critique de ses textes et de ceux des autres, enfin d'écrivain» (Dompmartin-Normand \& Le Groignec, 2015, p. 69). Enfin, l'écriture multilingue constitue une « révolution » au sein des institutions: «[...] les apprenantes, en utilisant leurs langues dans des textes en français, bousculent la norme du texte monolingue essentiellement pratiqué dans les centres d'apprentissage de langue française. » (Mathis, 2016, p. 266)

Les activités d'écriture multilingue ne sont pas réservées aux adultes. En effet, on constate leur développement en contexte scolaire, en particulier dans des classes accueillant un nombre important d'élèves primo-arrivants. Il s'agit alors de débutants en langue cible qui peuvent fréquenter la maternelle ou les classes du primaire. Les propositions d'écriture multilingue à ce niveau prennent des formes diverses : ce peuvent être des kamishibaï plurilingues ${ }^{2}$, des albums multilingues encore appelés «albums faits maison » (Sneddon, 2015, par exemple), des écrits multilingues produits à la suite de la lecture d'un écrit long ${ }^{3}$, ou encore une production écrite multilingue et multimodale numérique (Lotherington, 2015). Dans de nombreux cas, les parents ou membres de la communauté scolaire sont appelés à jouer un rôle d'expert pour la langue/culture d'origine auprès des enseignants. Il est primordial de noter que les propositions d'écriture multilingue s'appuient sur la langue de socialisation des enfants, à savoir la langue parlée à la maison et que les enseignants des classes n'hésitent pas à faire usage de la langue de l'apprenant, notamment à travers le translanguaging (Mary \& Young, 2017) pour construire les apprentissages en langue-cible.

En ce qui concerne les résultats obtenus dans ces démarches auprès d'enfants, Lotherington (2015) associe le développement d'espaces plurilingues dans des textes multimodaux à un « outil puissant pour l'apprentissage et la production de textes " (« $a$ powerful tool in both learning and text production ", p. 90), confortant ainsi les résultats mis en avant par Cummins en ce qui concerne les apports d'approches incorporant les langues d'origine pour le développement de la littératie (voir Cummins, 1981).

\section{Une démarche basée sur l'appropriation de supports hybrides}

Dans notre démarche, la thématique de l'exil a été retenue car elle concerne tous les apprenants. Sont abordés l'exil choisi (séjour d'étude, expatriation...) et l'exil forcé. Cette thématique permet aux apprenants du groupe de se dévoiler et à ceux dont les parcours sont les plus difficiles de partager leur expérience et d'évoquer leurs langues. Le corpus retenu est variét ${ }^{4}$ et vise à faire connaitre, de manière inductive, diverses manières d'écrire l'autobiographie et à aborder divers aspects de l'exil et de la migration. On espère à travers l'étude de ces textes, susciter, par effet de miroir, des textes pertinents sur l'expérience de l'exil et de la migration. Plusieurs types de textes sont proposés: des textes informatifs non littéraires (Lacoue-Labarthe et Schneider), des textes littéraires monomodaux (Houston, Thuy, Laferrière ${ }^{5}$, Bazin, Duras, ainsi que Adam, Mabanckou, Chagall et Cheng), des textes multilingues (Chraïbi ${ }^{6}$ et Plume ${ }^{7}$ ) et des iconotextes, à savoir, un texte autobiographique illustré (Stendhal), un album multilingue pour enfants 
(Godart) et une BD multilingue (Mahmoudi). Il est intéressant de noter que le caractère hybride de l'album et de la BD choisis tient à la fois à la co-présence texte-image mais aussi à la co-présence de langues différentes dans le texte, l'image ou dans les deux médiums. La pluralité des interactions observées en classe entre l'image et le texte (illustration, complémentarité, contradiction, analogie mais aussi métaphore, prolongement ou porosité) offre une palette d'outils pour l'écriture. À titre d'exemple, on peut observer que l'extrait de l'autobiographie de Stendhal, Vie de Henri Brulard (1927, édition originale 1890), a la particularité d'intégrer, en regard du texte, des dessins, schémas et croquis. Dans ce cas, les images ont une fonction illustrative. Elles ont aussi un rôle de complémentarité pour le lecteur qui est invité à pénétrer dans les lieux de l'enfance de manière visuelle. Pour ce qui est de la BD Arabico d'Halim Mahmoudi (2009), l'image apporte de nombreux éléments, en particulier pour se représenter le contexte culturel, le présent et le passé, l'ici et l'ailleurs. Les tableaux colorés représentant la vie passée en Algérie contrastent avec le noir, le blanc et le gris des images représentant la vie du personnage en banlieue parisienne. En outre, certaines images contredisent le texte, ou informent avec humour sur les pensées du personnage. L'album Maman Dlo d'Alex Godart (1998) est un album pour enfants multilingue, jalonné de mots en langue créole. Très riche sur le plan culturel, cet album illustre une situation d'abandon et d'exil aux Antilles ${ }^{8}$. L'image joue un rôle illustratif, mais elle ajoute aussi au texte, en convoyant les émotions notamment. L'image, parfois, contredit le texte.

Le corpus est complété par des témoignages réels, témoignages de gens ordinaires ${ }^{9}$. Ces exemples d'écriture de soi montrent que toute personne peut pratiquer l'écriture et produire des textes dignes d'intérêt, présentant des traits de littérarité, sans pour autant exercer le métier d'écrivain.

\section{Des objectifs pluriels}

Perfectionner le lire, continuer à se construire comme sujet lisant, mieux appréhender le genre autobiographique dans la lecture fait partie des objectifs de la démarche. Celle-ci vise également à s'approprier le genre autobiographique et à parfaire l'écriture dans un travail pouvant inclure l'image, le graphisme.

La démarche se doit par ailleurs de répondre aux besoins de tous les apprenants dans leur diversité pour que chacun progresse sur les plans linguistiques, littéraires, culturels et interculturels. Enfin, elle doit tendre vers une plus grande prise de conscience (" awareness ») en ce qui concerne le multilinguisme et l'expérience individuelle contemporaine des migrants.

Utiliser les langues de son répertoire est un procédé qui n'est pas imposé. Il prend sa place parmi d'autres. Il est envisagé dès le début comme une manière d'écrire, comme une expérience esthétique, mais aussi comme une manière de se dire, de dire ses langues, celles-ci étant perçues comme partie intégrante de l'identité de chaque apprenant.

Les quatre étapes essentielles de la démarche sont les suivantes : la lecture individuelle des textes et la préparation des cercles de lecture à la maison, le partage des lectures lors de cercles de lecture organisés en classe, les ateliers d'écriture en classe, et enfin la production d'un texte autobiographique individuel de 3000 mots. Cette production finale, évaluée en raison des exigences de l'institution, permet aux étudiants, s'ils le souhaitent, 
d'intégrer les textes (ou une partie des textes) qu'ils ont produits dans les ateliers d'écriture.

21 Les cercles de lecture, dispositif pédagogique retenu pour dialoguer sur les divers aspects des textes, permet aux apprenants d'établir des liens entre le fond et la forme ou encore de proposer des ponts avec des documents de leur choix.

Les propositions d'écriture sont toujours en lien avec les textes étudiés. On propose par exemple l'écriture de textes sur les plantes ou arbres du pays d'origine. Remarquons qu'aucun exercice portant spécifiquement sur l'usage des langues du répertoire de l'apprenant ou sur l'intégration de l'image n'a été proposé en raison des contraintes de temps ${ }^{10}$.

\section{Contexte et méthodologie}

23 L'institution où la démarche a été proposée est une université qui compte 25000 étudiants et est située en Australie-Méridionale. Nos cours généralement qualifiés « de français langue étrangère » accueillent des apprenants aux profils variés. Aux apprenants anglophones australiens ayant commencé leur apprentissage du français dans notre institution ou dans le secondaire s'ajoutent des étudiants internationaux originaires du Sud-Est asiatique ainsi que des réfugiés, nouvellement arrivés et venant de pays en guerre, pays d'Afrique francophone notamment. Ces derniers souhaitent faire reconnaitre leurs compétences en français. Dans la section de français et au niveau avancé, ces nouveaux migrants, majoritairement plurilingues, constituent une part non négligeable des effectifs (pour le groupe qui nous concerne, ils représentent $44 \%$ des inscrits).

Notre cours sur l'autobiographie et l'exil est proposé au niveau le plus avancé (cours de $4^{\mathrm{e}}$ année). Sur les neuf étudiants inscrits dans ce cours, cinq ont décidé de participer à la recherche. Sur ces cinq étudiants, deux sont plurilingues et francophones (originaire de la République démocratique du Congo pour l'un et du Liban pour l'autre), les trois autres étudiants apprennent la langue française comme langue étrangère. L'un de ces trois étudiants est en situation d'expatriation aux États-Unis et suit le cours à distance. Elle rejoint à la mi- semestre les discussions de classe via Skype. Le cours est un cours hebdomadaire de 2 heures sur un semestre (12 semaines). La recherche a été conduite après obtention de l'accord du comité d'éthique de notre université.

Les supports analysés ici sont :

- les productions écrites de 3000 mots finalisées en dehors de la classe ;

- les questionnaires remplis en fin de parcours.

26 Afin d'observer les traces de multilinguisme et autres traces d'hybridité présentes dans les productions, nous avons recours aux notions de " construction d'un rapport intime à l'écriture littéraire " (Demougin, 1998) et de «sémiographie des textes " définie par Moore (2012) comme « la mise en sens du texte par l'utilisation du dessin, du symbole, des couleurs, de l'espace et des outils de scription comme modes de symbolisation des informations »(p.63). On procède à l'analyse des réponses du questionnaire grâce à l'analyse de contenu selon Patton (2002). Nous croisons ensuite les résultats de l'analyse des productions écrites avec les réponses fournies par les apprenants au questionnaire distribué en fin de parcours. 
27 Le questionnaire intitulé «Fiche finale réflexivité » informe sur les profils de lecteur des apprenants (lecteur de littérature ou non), de scripteur (sujet écrivant? et si oui, de quel genre d'écrit ?). Il invite les apprenants à s'interroger à posteriori sur :

- la structure de leur texte ;

- leurs choix en matière d'écriture ;

- les textes qui les ont consciemment inspirés (textes du corpus ou lectures buissonnières) ;

- les passages de leurs textes où ils ont fait référence à ces œuvres ;

- les passages des textes lus qui les ont inspirés ;

- les techniques d'écriture dont ils pensent être à l'origine ;

- les apports de la démarche en termes de connaissance de soi.

\section{Métissage linguistique et autres traces d'hybridité dans les productions d'apprenants}

28 Il importe pour nous de voir tout d'abord si certaines formes d'hybridité telles que recours à l'image ou à des langues du répertoire ont été mobilisées dans ces productions et comment elles l'ont été. Nos questions de recherche sont les suivantes : le recours aux langues du répertoire est-il présent chez tous les apprenants y compris chez ceux dont les parcours ne sont pas ceux de migrants? Quelles formes le métissage linguistique prend-til ? Quelles sont les autres formes d'hybridité présentes et en particulier, l'apprenant a-til recours à l'image? Que dit l'apprenant quant aux textes ou iconotextes l'ayant influencé (stratégies narratives, reprise de thèmes...) ?

Mona, étudiante d'origine libanaise, propose un texte autobiographique riche et dense. Elle ne fait figurer aucun élément iconographique dans cette production, mais use d'une police d'écriture différente à un moment donné pour mettre en exergue un paragraphe d'analyse. Extrêmement fréquente dans l'album, cette technique a été observée dans la BD Arabico, dans l'album Maman Dlo ainsi que dans les textes multilingues d'Amélie Plume et de Driss Chraïbi. À deux reprises, elle fait figurer des termes arabes dans son texte. Elle choisit de faire apparaitre le terme arabe "Ya Binté » (« ma fille»), lorsque la narratrice relate sa conversation avec un vieux Libanais alors qu'elle s'apprête à quitter le Liban pour se marier en Australie: "Soyez heureuse Ya Binté : parce que notre Dieu vous a donné la chance pour découvrir sa belle création. C'est une grâce, soyez heureuse et profitez-en. » Elle fait aussi apparaitre le terme « khalsa » (" prête »), qu'elle intègre dans un paragraphe consacré à l'hospitalité libanaise. Le recours limité à l'arabe (2 occurrences) s'explique peut-être par le fait que pour cette apprenante, «Écrire en français, pour moi, fut toujours un moyen plus facile d'expression et de réflexion écrite bien que l'arabe soit ma première langue ». Partagée en classe, la production de Mona apporte un éclairage culturel très signifiant sur la francophonie. En présentant les prénoms (tous français) des membres de sa famille, Mona s'interroge pour savoir si ceuxci sont liés « au rôle culturel et politique qu'ont joué les Français au Liban dès le dixneuvième siècle jusqu'après la deuxième guerre mondiale ou au rôle de la tradition et de la foi chrétienne ». Ce faisant, son texte révèle aussi l'importance de la religion dans la vie et la culture de sa communauté d'origine. Autre élément intéressant pour le reste du groupe, Mona souligne la présence de gommiers au Liban, arbres apportés par les troupes australiennes pendant la Seconde Guerre mondiale. Mona entremêle donc les fils en lien avec la France, l'Australie (son pays d'adoption) et le Liban (son pays d'origine). Son texte présente d'autres aspects interculturels extrêmement intéressants pour la classe de 
français langue étrangère. Ainsi Mona met-elle en regard l'expérience douloureuse et traumatisante qu'elle a vécue en tant que jeune élève d'une école maronite avec celle, lumineuse et épanouissante, que vivent ses enfants en Australie.

Steve, apprenant australien qui se déclare non-lecteur de littérature mais scripteur de blogs sportifs en anglais, fait un retour dans son autobiographie sur son pays d'origine, l'Écosse. Il a recours à l'écossais dans le titre de la première partie de son texte, où il évoque le départ de sa famille pour l'Australie : «Soraidh le eilean cheo » — "Adieu à l'ile dans le brouillard». L'usage, certes limité, de la langue d'origine revêt cependant une importance qui dépasse la simple tonalité illustrative. En effet, en lisant la suite du texte de Steve, on mesure l'importance de cette langue et surtout d'un accent écossais qu'il n'a pas. Ayant, selon ses termes, "été enlevé à un jeune âge ", il fait aujourd'hui face à des interlocuteurs qui constatent qu'il n'a pas l'accent écossais. On devine implicitement le regret de ne pouvoir attester, par la présence de cet accent caractéristique, de ses origines et de son identité. Autre trace d'hybridité dans la production, l'ajout de photographies et d'une image. Dans le cas de Steve, l'image est une carte géographique. Cependant, celle-ci n'est pas une simple carte de son île. Elle a également une dimension décorative (esthétique) et culturelle, avec des visages ou symboles du pays présentés en médaillons. Notons ici qu'aucun des ouvrages iconographiques proposés en lecture n'utilisait d'illustration photographique ni de carte. Cependant, des cadres présentant des photographies de membres de la famille sont présents à la fois dans Maman Dlo et dans Arabico, lorsque sont représentés l'intimité familiale et l'intérieur de la maison.

Jane, apprenante australienne, en situation d'expatriation aux États-Unis, propose un texte sophistiqué, long et très construit. Celui-ci se présente sous forme de «leçons ». Une citation précède chacune des leçons. À travers cette technique, l'étudiante montre sa volonté de proposer une démarche narrative originale. La précision dans la langue et le nombre de textes soumis à correction avant la version finale montrent qu'elle s'est fortement investie dans son travail. Au niveau linguistique, on constate qu'il y a mélange des langues. L'étudiante a recours à l'anglais dans son texte lorsqu'elle utilise des termes dont elle ne connait pas l'équivalent direct en français comme, "Sunshine Coast » (côte orientale de l'Australie), "cling wrap» (qui désigne le film alimentaire plastique), " E10 poms ${ }^{11}$ ", «Royal Australian Air Force » (RAAF : l'armée de l'air australienne) ou le titre du film "An American in Paris ». L'intégration de ces termes "en version originale » s'accompagne le plus souvent d'une périphrase explicative en français. En outre, sa production intègre des photos accompagnant chaque partie de la vie relatée et d'une carte présentant le Canada et la région de Winnipeg, où elle a vécu pendant quelques années avec son mari. Les légendes qui accompagnent ces photos sont particulièrement intéressantes. En effet, la légende peut être un simple commentaire comme «moi, à 4 ou 5 ans ", mais elle peut aussi faire un clin d'œil au texte autobiographique et expliquer qui sont les personnes photographiées. La légende peut donc aussi avoir un caractère explicatif et ajouter des informations qui ne figurent pas dans le corps du texte. Ainsi, lorsqu'elle inclut une photo d'elle-même au sein d'un groupe de militaires à qui elle délivre une formation, elle précise en légende l'appellation du cours, les objectifs de celuici et la raison pour laquelle ses stagiaires ne portent pas l'uniforme. Enfin, elle indique sa position sur la photographie. À l'instar d'autres productions, telle que celle de Mona par exemple, son texte présente un intérêt interculturel pour les autres membres du groupe. Dans son cas, on pense au partage de son expérience passée d'expatriée australienne dans une région de grand froid (le centre du Canada). On pense encore au 
récit de l'histoire familiale qui a conduit ses grands-parents à quitter l'Angleterre pour venir s'installer en Australie ${ }^{12}$.

Katy, apprenante australienne monolingue, n'a pas recours dans son texte à une langue autre que le français. Son texte prend la forme d'une lettre qu'elle adresse à un lecteur fictif. Celui-ci en prendra connaissance après le décès de son auteure. Dans cette lettre, l'apprenante relate ses voyages et sa découverte de la France et de l'Angleterre après avoir présenté sa famille et sa petite ville d'origine du sud de l'Australie. Son texte est accompagné de nombreuses photographies de voyage et de photographies qui marquent son attachement à ses racines australiennes et à sa famille. Faisant écho à l'injonction adressée à son lecteur de partir découvrir d'autres pays sans toutefois oublier d'où il vient, les photographies de l'Europe et des membres de la famille cèdent la place dans la toute dernière partie de sa production à quelques photographies exotiques d'un voyage récent en Indonésie. De manière intéressante, et à l'instar d'observations faites lors de la lecture des albums pour la jeunesse, les photographies choisies ne se contentent pas d'illustrer le texte. Si une photographie de la tour Eiffel est bien placée en regard du texte décrivant l'émotion de l'auteure à la vue de ce monument, l'apprenante ajoute également quelques photographies prises en Italie alors qu'il n'est pas question de ce pays dans son texte.

Roland est un apprenant originaire d'Afrique francophone nouvellement arrivé en Australie. Bien que plurilingue (il parle 6 langues : le français, l'anglais et quatre langues d'Afrique centrale, sa région d'origine), Roland n'a pas utilisé les langues de son répertoire dans son texte autobiographique, à l'exception de l'expression anglaise " fruitpicking " utilisée dans le contexte de la cueillette des fruits en Australie-Méridionale. Il aurait été intéressant de savoir pourquoi Roland n'a pas inclus de langue de son répertoire dans sa production. Malheureusement, en raison des règles du comité d'éthique de notre institution, nous n'avons eu accès à la production finale de cet étudiant qu'une fois le semestre terminé et n'avons pu contacter cet apprenant par la suite. Néanmoins, nous nous posons les questions suivantes quant à la non-intégration des langues de son répertoire : était-ce quelque chose qui, selon lui, « ne se faisait pas »? Est-ce parce qu'il considère qu'une telle pratique entrave la lecture ? Ou bien encore estce parce qu'il est d'avis que les langues minoritaires qu'il parle sont moins légitimes que le français, langue de scolarisation dans son pays d'origine? Est-ce, enfin, parce que ces langues y sont utilisées essentiellement oralement et non à l'écrit?

Bien que non porteur de langues du répertoire, son texte est néanmoins porteur de variations, ce qui contribue à sa portée culturelle, et témoigne de la richesse langagière de cet apprenant. En écrivant "la musique tapait record dans ce salon de coiffure", lorsqu'il évoque le retour au pays natal et les retrouvailles avec ses amis dans ce lieu chargé de souvenirs, cet apprenant s'est autorisé à utiliser en français des expressions propres au pays d'origine, des variations francophones. Peut-être l'approche proposée at-elle contribué à ce résultat, alors que, comme le souligne Moïse (2007), l'insécurité linguistique est forte dans les pays francophones et «la langue française, marquée du poids de ses idées, et sous l'emprise d'une norme objective ou imaginée, induit non seulement un bien parler, mais un bien écrire » (p. 310).

Pour ce qui est des autres apprenants du groupe, on remarque que deux d'entre eux proposent des productions très intéressantes. L'un d'eux, un étudiant australien ayant des origines à la fois européennes et aborigènes, est inscrit dans la filière cinéma. Il intègre de manière judicieuse une partie du script de film qu'il a écrit pour un de ses 
autres cours dans son texte autobiographique. Son texte est donc un entrelacs entre un texte autobiographique rédigé à la première personne du singulier et un texte fictif de genre cinématographique, dont la police de caractère est celle d'un texte dactylographié. Ce script de film dénonce les violences faites aux aborigènes. Deux termes en anglais apparaissent dans son texte. Il s'agit de termes utilisés pour désigner de manière raciste les aborigènes ou ceux dont le sang est mêlé. L'engagement de l'apprenant dans sa production se mesure à travers le travail d'écriture, mais aussi à travers l'effort de traduction d'extraits de son script de film.

Autre production intéressante, celle d'un jeune étudiant australien, qui mêle photographies, coupure de journal, reproduction d'un courriel bilingue et de la couverture du manuel d'entretien de sa première voiture. Cet apprenant qui affirme «écrire des textes sans émotion » de type instructions ou essais dans sa langue première, intègre très judicieusement le courriel bilingue dans lequel sa grand-mère l'encourage avec humour et amour à poursuivre ses études de français. Il affirme que cette démarche lui a été inspirée par les textes et iconotextes lus.

\section{Discussion}

Dans notre contexte particulier, il importait de proposer une approche inclusive qui permette à chacun de voir ses compétences, son identité et ses langues reconnues et valorisées. Pour ceux dont les parcours étaient moins complexes et moins douloureux, l'approche se devait de constituer une ouverture vers l'autre, vers l'apprenant aux identités et aux langues multiples, et participait d'une démarche de justice sociale. Cette expérience, qui a révélé à la classe les expériences et parcours plurilingues souvent masqués à l'université, a mis en avant le rapport aux langues des individus, des communautés, et mis en lumière des situations de diglossie.

Les textes de caractère rétrospectif/introspectif ont permis sur le plan interculturel d'obtenir des échanges de grande qualité qui ont constitué une ouverture sur les pays de langue française et le reste du monde. Grâce à cette démarche, des liens entre plurilingues et non-plurilingues ont été tissés, favorisant ainsi une meilleure connaissance de l'autre au sein du groupe. L'enseignant n'était plus dans un rôle de transmetteur d'informations, mais de relais et de modérateur. Enfin, notre démarche a contribué à répondre aux objectifs d'internationalisation fixés par l'institution.

39 À l'intérieur du corpus sélectionné, l'hybridité prenait plusieurs formes : tantôt hybridité linguistique, lorsqu'elle mêlait le français à d'autres langues du répertoire du scripteur, tantôt hybridité modale lorsque l'image était présente. Dans certains cas, l'hybridité tenait à la fois au mélange des langues du répertoire et de l'image. On pourrait encore évoquer l'usage d'une police d'écriture différente comme caractéristique additionnelle d'hybridité. Participant de l'esthétique particulière des textes produits, ces caractéristiques ont été observées dans les textes d'apprenants, montrant ainsi qu'ils s'étaient appropriés les techniques observées chez les auteurs étudiés ou avaient essayé de proposer des démarches originales. Pour autant, le répertoire linguistique des apprenants n'a peut-être pas été exploité autant qu'il aurait pu l'être. Des phases spécifiquement dédiées à l'écriture multilingue pourraient être envisagées afin d'inciter les apprenants à intégrer de manière plus prégnante les langues de leur répertoire dans leurs écrits. En outre, les apports des supports multimodaux multilingues doivent faire l'objet de plus amples recherches, tout comme le recours à divers logiciels d'écriture. 
Pour nous, l'image qui «ne se contente pas de représenter les choses, [mais] les montre inscrites dans un contexte » (Le Manchec, 2006, p. 154), a un rôle crucial à jouer, au même titre que « les langues en réserve».

\section{BIBLIOGRAPHIE}

BARONI, Raphaël \& BEMPORAD, Chiara. (2011). Exploitation de la démarche biographique en classe de langue. A contrario, 15, 117-133.

BISHOP, Marie-Françoise \& PENLOUP, Marie-Christine. (2006). L'écriture de soi et l'école : une relation singulière. Repères, 34, 5-11.

CARDELl, Kylie \& Douglas, Kate. (2018). Why Literature Students Should Practise Life Writing [2016]. Arts \& Humanities in Higher Education, 17(2), 204-221. <https:// doi.org/10.1177/1474022216635825>.

ChATEAuReynaud, Marie-Anne \& OROZ-Aguerre, Maiana. (2016, novembre). Aventure littéraire plurilingue en contexte scolaire frontalier. Communication présentée lors du colloque « Livre ensemble », Université Toulouse-Jean Jaurès. Disponible en ligne sur <www.canal-u.tv/ producteurs/universite_toulouse_ii_le_mirail/colloques/livre_ensemble> (consulté le 10 janvier 2018).

CROZET, Chantal. (2014, juillet). Biographical Writing in the French Classroom. Communication présentée au colloque «Vivre le français au XXI ${ }^{\mathrm{e}}$ siècle : transition, innovation, collaboration » de l'AFTV/FATFA, Melbourne, Australie.

Cummins, Jim. (1981). Bilingualism and Minority Language Children. Toronto : Ontario Institute for Studies in Education of the University of Toronto.

Cummins, Jim \& EARLY, Margaret (dir.). (2011). Identity Texts: The Collaborative Creation of Power in Multilingual Schools. Londres : Institute of Education Press.

Demougin, Patrick. (1998). Littérature et pratique d'écriture en FLE : clarifier les enjeux. Travaux de didactique du FLE, 39, 69-77.

Diallo, Ibrahima \& Maizonniaux, Christèle. (2017). Policies on Pedagogy for Students of Diverse Backgrounds. International Journal of Pedagogies and Learning, 11(3), 202-210.

Dompmartin-Normand, Chantal \& LE Groignec, Anne. (2015). Un atelier d'écriture créative en FLE. Tissages et apprentissages plurilingues. Éducation et sociétés plurilingues, 38, 59-71. Disponible en ligne sur <http://journals.openedition.org/esp/539>.

EDWARDS, Nathalie \& HOGARTH, Christopher. (2016). Autobiography in the Language Classroom. Auto/Biography Studies, 32(1), 55-65.

GEY, Catherine \& CARLADOUS, Alice. (2016). Production d'écrits en classe UPEAA : le kamishibaï plurilingue. Communication présentée lors du colloque «Livre ensemble ", Université ToulouseJean Jaurès. Disponible en ligne sur <www.canal-u.tv/producteurs/ universite_toulouse_ii_le_mirail/colloques/livre_ensemble> (consulté le 10 janvier 2018). 
GREAVES, Sara \& Di STEPHANO, Jean-Luc. (2015). Atelier d'écriture plurilingue sur l'exil. Disponible en ligne sur <https://f-origin.hypotheses.org/wp-content/blogs.dir/830/files/2016/03/Atelierexil-1-tous-usages-S.-Greaves.pdf> (consulté le 11 juin 2017).

LEJEUnE, Philippe. (2001). Enseigner à écrire l'autobiographie. Dans M.-H. Roques (dir.), L'autobiographie en classe (p. 13-22). Toulouse : Delagrave Éditions et CDPR, Midi-Pyrénées.

LE MANCHEC, Claude. (2006). L'écriture de soi dans la littérature de jeunesse : description et enjeux didactiques. Repères, 34, 141-164.

LIYANAGE, Indika, SINGH, Parlo \& WALKER, Tony. (2016). Ethnolinguistic Diversity within Australian Schools: Call for a Participant Perspective in Teacher Learning. International Journal of Pedagogies and Learning, 11(3), 211-224.

LOTHERINGTON, Heather. (2015). DIY Plurilingual Literature. A Multimodal Approach to Linguistic Inclusion in the Urban Elementary Classroom. Dans C. Hélot, R. Sneddon \& N. Daly (dir.), Children's Literature in Multilingual Classrooms: From Multiliteracy to Multimodality (p. 84-103). Londres : Institute of Education / Trentham Books.

MaIzonniaux, Christèle. (À paraitre). Littérature de jeunesse et écritures créatives en classe de langue : un parcours en contexte universitaire australien.

MAIzONNIAUX, Christèle \& HANSON, Joanne. (À paraitre). A Foreign Language Learner's Journey with (into) Life Writing.

MARY, Latisha \& Young, Andrea. (2017). From Silencing to Translanguaging: Turning the Tide to Support Emergent Bilinguals in Transition from Home to Pre-School. Dans B. Paulsrud, J. Rosen, B. Straszer \& A. Wedin (dir.), New Perspectives on Translanguaging and Education (p. 108-128). Bristol : Multilingual Matters.

Mathis, Noëlle. (2014). Pratiques de littératies en atelier d'écriture plurielle : une fenêtre ouverte à l'expression d'identités plurilingues. Spirale, 53, 73-85.

MATHIS, Noëlle. (2016). Écrire (entre) ses langues en atelier d'écriture. Une approche plurilingue des textes de Driss Chraïb, Wadji Mouawad et Nancy Huston. Carnets : revue électronique d'études françaises, II(7), 251-267. Disponible en ligne sur <http://ler.letras.up.pt/uploads/ ficheiros/14217.pdf> (consulté le 13 février 2018).

MoÏsE, Claudine. (2007). Variations et créations ou d'inévitables rencontres interculturelles. Études de linguistique appliquée, 147, 307-316. Disponible en ligne sur <http://cairn.info/revueela-2007-3-page-307.htm> (consulté le 28 aout 2017).

MOORE, Danièle. (2006). Plurilinguismes et école. Paris : Didier.

MOORE, Danièle. (2012). Pratiques plurigraphiées d'enfants chinois en immersion française à Vancouver. Recherches et Applications, 51, 62-75.

MONTELLE, Edith. (2007). La boîte magique, le théâtre d'image ou kamishibaï : histoire, utilisation, perspectives. Strasbourg: Callicéphale éditions.

PATTON, Michael Quinn. (2002). Qualitative Research and Evaluation Methods (3 $3^{\mathrm{e}}$ éd.). Thousand Oaks : Sage.

Schultze, Marie-Laure. (2014, février). Ateliers universitaires d'écriture plurilingue - qui (d'où), pourquoi, comment et vers quoi? Pour une nouvelle didactique. Communication présentée au colloque international « Writing Research Across Borders (WRAB) III », Université Paris-Ouest Nanterre La Défense, France. 
SNEDDon, Raymonde. (2015). Reading and Making Books in Two Languages. Dans C. Hélot, R. Sneddon \& N. Daly (dir.), Children's Literature in Multilingual Classrooms: From Multiliteracy to Multimodality (p. 121-137). Londres : Institute of Education / Trentham Books.

UniVERSITÉ TOUlouSE-JEAN JAURÈs (Toulouse 2-Le Mirail). (2016, novembre). Colloque intitulé «Livre ensemble : pluriculturalisme et plurilinguisme à travers les albums pour la jeunesse». Disponible en ligne sur <https://www.canal-u.tv/producteurs/universite_toulouse_ii_le_mirail/ colloques/livre_ensemble> (consulté le 28 aout 2017).

NB. - On trouvera un certain nombre des interventions de ce colloque sous forme d'articles dans un numéro à paraitre de la revue Strenae, disponible prochainement en ligne sur <https:// strenae.revues.org/1680>.

\section{ANNEXES}

\section{Corpus d'extraits de textes littéraires et d'iconotextes proposés à l'étude.}

ADAM, Olivier. (2009). Extrait de «A l'abri de rien». Dans L. Barbizet-Namer (dir). Nouvelles Odyssées. 50 écrivains racontent l'immigration. Paris : éditions de la Cité nationale de l'histoire de 1'immigration. 50-51.

BAzIN, Hervé. (2010. [Sans titre]. Dans M. Bisiaux et C. Jajolet (dir). A ma mère. 50 écrivains parlent de leur mère. Paris: Horay. 23-27.

Chagall, Marc. (2009). Extrait de «Ma vie». Dans L. Barbizet-Namer (dir). Nouvelles Odyssées. 50 écrivains racontent l'immigration. Paris : éditions de la Cité nationale de l'histoire de 1'immigration. 167-168.

CHENG, François. (2009). Extrait de « Le dialogue ». Dans L. Barbizet-Namer (dir). Nouvelles Odyssées. 50 écrivains racontent l'immigration. Paris : éditions de la Cité nationale de l'histoire de 1 'immigration. 163-164.

CHRAIIBI, Driss. (1998). Vu, lu, entendu. Paris : Denoë1. 28-29.

DURAS, Marguerite. (2010). [Sans titre]. Dans M. Bisiaux et C. Jajolet (dir). A ma mère. 50 écrivains parlent de leur mère. Paris: Horay. 135-141.

GODART, Alex. (1998) Maman Dlo. Paris : Albin Michel Jeunesse.

Houston, Nancy \& SEBBAR, Leila. (1999). Lettres parisiennes. Autopsie de l'exil. Paris : J'ai lu. $117-118$.

LACOUE-LABARTHE, Isabelle. (2013). 'De l'exil forcé à l'exil choisi... entretien avec Elias

Sanbar'. Diasporas, 22, 21-37.

LAFERRIERE, Dany. (2012). Chronique de la dérive douce (2e édition). Montréal : Les Éditions

Boréales. 205-209.

MABANCKOU, Alain. (2009). Extrait de «Bleu, blanc, rouge ». Dans L. Barbizet-Namer (dir).

Nouvelles Odyssées. 50 écrivains racontent l'immigration. Paris : éditions de la Cité nationale de

l'histoire de l'immigration. 89-91.

MAHMOUd, Halim. (2009). Arabico. T1: Liberté. Paris : Éditions Soleil (Quadrants). 1-11.

PLUME, Amélie. (1998). Ailleurs, c'est mieux qu'ici. Carouge-Genève : Éditions Zoé. 143-145.

SCHNEIDER, Anne. (2013). La littérature de jeunesse migrante. Paris : L'Harmattan. 17-19.

SEWTOwUr, Amal. (2012). Made in Mauritius. Paris : Gallimard . 24-45.

STENDHAL. (1927). Vie de Henri Brulard. Paris : Le divan. 1-19 et 42-59.

THUY, Kim. (2010). Ru. Paris: Le Livre de Poche. 11-19.

\section{NOTES}

1. Notion développée par Cummins et Early (2011).

2. Le kamishibaï est une technique de contage d'origine japonaise. Les artistes utilisent des planches cartonnées présentant d'un côté le texte (qui fait face au lecteur) et de l'autre l'image (telle un tableau qui fait face au spectateur). À partir de 1923, apparaissent les premiers kamishibaïs pour enfants. Dispositif léger et peu onéreux, il est utilisé pour l'alphabétisation, la lecture à haute voix et la lecture de l'image ainsi que pour inviter des enfants à créer des histoires. Pour plus d'informations à ce sujet, se référer à Montelle (2007). Pour ce qui est de 
l'élaboration de kamishibaïs multilingues en classe, on pourra se référer à Gey et Carladous (2016).

3. On pense ici à Chateaureynaud et Oroz-Aguerre (2016).

4. La liste des ouvrages et textes étudiés est présentée en annexe.

5. Je remercie M. Deraîche pour cette référence ainsi que pour m'avoir indiqué l'ouvrage de Kim Thuy.

6. Il s'agit d'un extrait de $V u$, lu, entendu (1998). On pourra se référer à l'analyse très pertinente des formes que prend le multilinguisme dans cet extrait dans Mathis (2016).

7. Je remercie ici M.-L. Schultze et N. Mathis pour ces références.

8. L'album présente une fillette antillaise vivant chez ses grands-parents après le départ de sa mère partie travailler en métropole. L'album fait référence à une réalité socioculturelle (la place des filles dans la société antillaise, le respect dû aux adultes et aux anciens, l'analphabétisme des grands-parents, la pauvreté, l'exil maternel, l'isolement) et intègre des aspects patrimoniaux avec le récit enchâssé de la légende des sirènes (les «mamans dlo ») qui attirent au fond de la mer ceux qui succombent aux illusions de la richesse.

9. A été suggérée la lecture de textes présentés sur le site <http://raconterlavie.fr/> (remplacé désormais par <http://raconterletravail.fr/projet/> (consulté le 11 aout 2017) ainsi que sur < www.francparler-oif.org/1990-2015-25-ans-25-textes-de-lafrique-francophone-au-sud-dusahara-et-de-locean-indien/> (consulté le 11 aout 2017).

10. L'intégration de ce point de vue méta fait cependant partie d'un nouveau projet de recherche actuellement en cours.

11. Selon Jane, «l'expression "Pom" ou "Pommy" est une abréviation du mot "pomegranate", une grenade, qui décrit la couleur rouge de la peau des immigrés anglais, brûlée facilement par le soleil australien rude ».

12. Le texte de Jane fait l'objet d'une analyse détaillée dans un article à paraitre (Maizonniaux \& Hanson, à paraitre).

\section{RÉSUMÉS}

On constate ces dernières années l'émergence de travaux consacrés à l'écriture multilingue dans le cadre d'ateliers d'écriture universitaires, ou, en contexte scolaire, de travaux invitant à l'écriture après découverte de livres pour la jeunesse hybrides. La démarche exploratoire présentée ici concerne des apprenants de français de niveau avancé d'une université australienne. L'analyse porte sur des textes autobiographiques écrits après avoir lu des ouvrages sur le thème de l'exil. Elle révèle le caractère protéiforme des productions d'apprenants ainsi que les potentialités offertes par l'inclusion de supports iconotextuels multilingues pour l'écriture.

In recent years, there has been a notable emergence of publications devoted to multilingual writing in the context of university-level writing workshops or, in the school context, on activities to encourage writing following the exploration of hybrid children's books. The exploratory approach presented here involves advanced-level students of French at an Australian university. The analysis focuses on autobiographical texts written after reading works on the theme of exile. It reveals the multifaceted nature of the students' writing as well as the potential afforded by the inclusion of multilingual iconotextual material intended to stimulate writing. 
INDEX

Mots-clés : autobiographie, migration, écriture multilingue, livres pour enfants, expérience littéraire et esthétique, multimodalité

Keywords : autobiography, migration, multilingual writing, children's books, literary and aesthetic experience, multimodality

\section{AUTEUR}

\section{CHRISTÈLE MAIZONNIAUX}

Flinders University \& UMR 5316 Litt\&Arts, Université Grenoble Alpes 